\title{
Plan de alta continuidad del cuidado en la consultoría de enfermería neurológica
}

\author{
Ma. Guadalupe Nava Galán*
}

\begin{abstract}
RESUMEN
El plan de alta de enfermería es un documento que proporciona información importante e individualizada al paciente y cuidador primario (familiar: padres, esposa o hijos); es elaborado por el enfermero que atiende al paciente durante su hospitalización. Este documento informa y favorece la continuidad de los cuidados a partir de su hospitalización hasta el egreso hospitalario, permite la comunicación entre los profesionales de enfermería en las distintas áreas clínicas de asistencia, ya sea primaria o especializada para proporcionar cuidado de calidad y acorde a cada paciente. Objetivo: Proporcionar información individualizada mediante el plan de alta para el paciente y cuidador primario, facilitar la continuidad del cuidado en el hogar y dar seguimiento a éste en la consultoría de enfermería neurológica. Se lleva a cabo mediante el proceso enfermero: consiste en valorar, en cada enfermo, las necesidades para establecer una adecuada planeación desde el ingreso hasta el egreso. Conclusiones: El plan de alta debe comunicarse con antelación al paciente y familia, es un apoyo didáctico para garantizar el proceso de los cuidados, y la coordinación interprofesional va a facilitar la comunicación para resolver dudas acerca de los cuidados que se van a proporcionar al enfermo, facilita y evita complicaciones durante la administración de la medicación, higiene personal o cuidados a la piel, disminuye los errores y es de gran ayuda.
\end{abstract}

Palabras clave: Plan de alta, consultoría de enfermería, cuidado.

\section{Discharge plan continuity of care in neurological nursing consultancy}

\begin{abstract}
The nursing discharge plan is a document that provides important information and individualized to the patient and primary caregiver (family: parents, wife or children), is prepared by the nurse has attended the patient during hospitalization. This paper reports and promotes continuity of care from hospitalization until hospital discharge, allows communication between nurses in different clinical areas of assistance, whether primary or specialized to provide quality and responsive care to each patient. Lens: Provide individualized by the discharge plan for the patient and primary caregiver facilitates continuity of care at home and follow this in neurological nursing consulting. It is performed by the nursing process: is to assess each patient needs to establish adequate planning from admission to discharge. Conclusions: The discharge plan should be communicated in advance to the patient and family, is a didactic support to ensure continuity of care and coordination will facilitate interprofessional communication to resolve doubts about the care to be provided to the patient, the easier and avoids complications during the administration of medication, personal hygiene or skin care, reduces errors and is of great help.
\end{abstract}

Key words: Discharge planning, consulting nursing, care.

* Maestría en Enfermería en Educación, Coordinadora de Investigación en Enfermería. Instituto Nacional de Neurología y Neurocirugía.

Correspondencia: Ma. Guadalupe Nava Galán. Insurgentes Sur Núm. 3877, 14269, La Fama, Del. Tlalpan, México, D. F.

E-mail: magpengalan@hotmail.com

Este artículo puede ser consultado en versión completa en http://www.medigraphic.com/enfermerianeurologica 


\section{INTRODUCCIÓN}

\section{Antecedentes}

$\mathrm{E}$ n la década de los 80 el Plan de Alta (PA) hospitalaria era percibido como sinónimo de suceso, misión cumplida o final triunfante de una serie de sofisticados cuidados médicos y de enfermería; no obstante, en caso de haber existido un gran número de pacientes correctamente capacitados, no hubieran reingresado al hospital por complicaciones que podrían ser prevenidas o controladas desde el hospital, sobre todo, en el hogar.

Diferentes enfoques reconocen que el PA es una herramienta útil en el proceso de transmisión de la responsabilidad del cuidado a familiar y cuidador primario, así como para otros profesionales de salud; el profesional de enfermería considera que en él se implementa el proceso enfermero a través de la valoración de necesidades, diagnósticos oportunos, plan de cuidados específicos y personalizados, sobre todo especializados, éstos son ejecutados y evaluados de manera decidida durante la hospitalización y brindan constancia del cuidado en el hogar.

La responsabilidad del profesional de enfermería es proporcionar capacitación, generar confianza y seguridad al cuidador primario de acuerdo con el diagnóstico y requerimientos de cada enfermo; de esta manera se favorece la calidad del cuidado. Girard manifiesta que a los enfermos y familiares hay que enseñarles y motivarlos para llevar a cabo el autocuidado desde que el profesional de enfermería los atiende por primera vez.

El PA consiste en una serie de recomendaciones que se le dan al paciente en forma oral y escrita, de tal manera que sean comprendidas a cabalidad una vez que el médico ha notificado el alta y se han llevado a cabo los trámites previos a ésta. Cabe destacar que las indicaciones, tanto orales como escritas, deben ser comprensibles y claras por parte del profesional de enfermería, verificando siempre la forma como el mensaje ha sido captado por el enfermo y su familiar o cuidador.

"La enfermería es una profesión liberal y una disciplina de carácter social, cuyos sujetos de atención son la persona, la familia y la comunidad, con sus características socioculturales, sus necesidades y derechos, así como el ambiente físico y social que influye en la salud y el bienestar. El ejercicio de la enfermería tiene como propósito general promover la salud, prevenir la enfermedad, intervenir en el tratamiento, rehabilitación y recuperación de la salud, aliviar el dolor, proporcionar medidas de bienestar y contribuir a una vida digna de la persona [...]". 5

Existen programas de enfermería de la Universidad de Caldas, en Colombia, que lo llevan a cabo a través de planes de alta a partir de la nemotecnia: MÉTODO. Éste corresponde a: Medicamentos, Entorno, Tratamientos no farmacológicos, Organización ambulatoria, Dieta y Otros.

La finalidad es reforzar y ampliar los cuidados que debe tener la persona enferma durante la convalecencia; éstos son proporcionados por ella misma (autocuidado) o por su familia o cuidador (asistencia); se proponen nuevos aspectos para incluir en el plan de alta. La Nursing Outcomes Classification (NOC) incluye varios de los aspectos necesarios para que la persona que asiste al enfermo se pueda desempeñar de manera satisfactoria durante su rol, de manera similar son igualmente importantes cuando quien proporciona los cuidados es la persona misma.

Pinzón propone que para facilitar su memorización y no pasar por alto algún aspecto básico, se utilice la siguiente nemotecnia: CUIDARME, que hace alusión al objeto de estudio de la profesión, es decir, el "cuidado", pero también a la responsabilidad que tenemos todas las personas frente a nuestra salud. Esta nemotecnia corresponde a: Comunicación, Urgente, Información, Dieta, Ambiente, Recreación y uso del tiempo libre, Medicamentos y tratamientos, Espiritualidad.

Actualmente, la Organización Mundial de la Salud (OMS) ha desarrollado "Nueve soluciones para la seguridad del paciente", a fin de ayudar a reducir daños relacionados con la atención sanitaria en todo el mundo. Las soluciones han sido formuladas sobre la seguridad del paciente. Cada uno de estos aspectos resalta la necesidad de adaptarlos a cada persona en particular, teniendo en cuenta sus características y necesidades, de tal manera que se proporcione un cuidado individualizado e integral.

Comunicación efectiva: Actualmente es una meta internacional propuesta para identificar a la persona a la cual se atiende en la institución; el equipo de salud debe acudir al enfermo cuando presente molestias o cuando se requiera, para evitar complicaciones, y con su familia para solicitar colaboración; ésta es una red de apoyo fundamental para la aplicación del plan de alta.

Urgente: Informar acerca de los signos de alerta que indican un deterioro de salud del paciente o de una recaída, datos que la persona enferma debe conocer, así como las acciones que debe llevar a cabo si se presentan; de este punto depende, en buena medida, la calidad de vida de la persona enferma; se debe incluir información sobre sitio, fecha y hora del próximo control para llevar un adecuado seguimiento; en este caso es la consultoría médica y de enfermería.

Información: El médico tratante es quien la proporciona al enfermo y su familia, la información es sobre tratamiento y pronóstico; el profesional de enfermería informa al paciente sobre los cuidados que se van a realizar para favorecer su autonomía, respeto y trato digno; tiene prohibido hacer 
pronósticos o evaluaciones con relación a los diagnósticos o tratamientos; enfermería informa sobre las intervenciones, es quien aclara las dudas que tenga la persona respecto a los cuidados, e indaga si requiere alguna otra información sobre su estado clínico; debido a la cercanía, se encarga de identificar oportunamente necesidades o vacíos en el conocimiento de la enfermedad que tiene el paciente y el familiar, así como verificar si conoce sus derechos relacionados con la salud, cómo y dónde ejercerlos.

Dieta: Este aspecto incluye la información relativa a dietas especiales, si las requiere, educación e información sobre alimentación balanceada según el ciclo vital, higiene y conservación de alimentos, horarios de alimentación y otros temas relacionados, de acuerdo con los datos que se hayan obtenido al valorar el patrón nutricional y metabólico; deben tenerse en cuenta los gustos y la capacidad económica de la persona para sugerir alimentos que estén a su alcance y para facilitar la adopción de cambios que redunden en un mejor nivel de salud.

Ambiente: Para hacer las recomendaciones acerca de este punto, es necesario haber indagado previamente sobre el ambiente doméstico, laboral y social en el que se desenvuelve la persona para incidir en su salud y capacitar a su familia para proporcionar educación e información correspondientes. Entre otros aspectos sugiere manejo de basura, higiene del hogar, posesión de animales domésticos y plantas (por la posibilidad de zoonosis que desencadene crisis o problemas alérgicos, entre otros); o bien riesgos que puedan ocasionar caídas, ubicación de la habitación respecto de otras áreas a donde debe desplazarse (baño, por ejemplo), en especial si la persona tiene alguna limitación para la movilización o un deterioro visual; o en relación con la ventilación e iluminación de la vivienda y del sitio de trabajo, uso de elementos de protección laboral. Se pretende considerar aspectos del ambiente que rodean al enfermo, que sean relevantes de acuerdo con su estado de salud, sobre todo alertarlo sobre los factores de riesgo y los factores protectores.

Recreación y uso del tiempo libre: Se deben de tener en cuenta los datos recolectados durante la valoración, para sugerir a la persona cómo implementar su tiempo libre, de acuerdo con ello brindar información y educación que apunten a prácticas recreativas saludables, al fortalecimiento de los nexos familiares, sociales, de pareja y de amistad. Es conveniente comentarle o sugerirle sobre los recursos que existen cercanos al lugar de la vivienda o trabajo, los cuales podrían resultar atractivos y adecuados a las condiciones de la persona, como lugares de recreación que proporcione la delegación cercana para poder participar en grupos de apoyo donde tenga actividades recreativas como caminatas, sugerirle que la actividad física es necesaria para la salud. Es importante que tenga en cuenta la socialización a través de salidas, paseos, recibir y hacer visitas, siempre y cuando esto no interfiera o constituya un riesgo para la persona.

Medicamentos y tratamientos farmacológicos: Transcribir con letra clara para saber cómo continuar su medicación en casa adaptando el horario más adecuado para la persona, teniendo en cuenta su ritmo de vida y las características de los medicamentos; por ejemplo, que algunos no deben administrarse en forma simultánea con otros, que las comidas pueden interferir con la absorción de algunos de ellos; en cambio, para otros se recomienda la administración con los alimentos. Se le debe informar que las reacciones adversas de unos cuantos pueden interferir con algunas actividades cotidianas, entre otros aspectos a considerar.

Sobre todo se debe tener en cuenta si la persona sabe leer o si comprendió la información para llevar a cabo su tratamiento, o bien si se deben generar estrategias a través de símbolos o si se le debe guiar con algún indicio que lo alerte sobre el uso seguro de la medicación. Se debe hacer hincapié en la duración del tratamiento, en especial cuando se trata de enfermedades crónicas que requieren medicación durante toda la vida. Informar y escribir con claridad aquellos signos que indican reacciones adversas a los medicamentos y que exigen consulta de inmediato, considerando reacciones adversas como: náuseas y las alteraciones de la frecuencia cardiaca con los digitálicos, las hemorragias al recibir anticoagulantes. Si es el caso, tratar explícitamente los riesgos de la automedicación y la importancia de acudir a la asistencia profesional en caso de requerirse.

No olvidar que, con frecuencia, las personas consumen medicamentos para patologías diferentes a lo que motivó la consulta: a menos que el médico indique lo contrario los medicamentos que deben seguir administrándose situación que debe ser comentada al enfermo o a su cuidador.

Incluir información sobre centros de salud o clínicas para dar continuidad y vigilancia a tratamientos que requiera la persona cuando se encuentre en lugares lejanos a la institución, donde realicen curaciones o vigilancia de escaras (frecuencia, sitio al cual debe acudir), cambios de sonda, administración de oxígeno, nebulizaciones, actividades recomendadas y desaconsejadas o "prohibidas", tiempo que debe esperar antes de reiniciar su vida sexual y modificaciones sugeridas a ésta de acuerdo con la situación clínica y la evolución de la persona (actividades que exigen un esfuerzo físico); indicación acerca de los periodos de reposo recomendados de acuerdo con su estado, en particular en algunos postoperatorios e incluso en algunas patologías médicas, técnicas para manejo del estrés, ejercicios, fisioterapia, entre otros.

En ocasiones, y en especial cuando la persona debe convivir por largo tiempo con tratamientos específicos que ameritan un entrenamiento previo, de tal manera que cuando sea 
dada de alta, su cuidador primario esté capacitado correctamente para realizar cuidados específicos a traqueotomía, aspiración de secreciones, ministración de nebulizaciones y oxígeno, gastrostomía permanente; manejo adecuado de sonda vesical, manejo de diálisis para evitar infecciones, entre otros.

Espiritualidad: La espiritualidad es una parte de la totalidad humana que "agrupa ideas filosóficas acerca de la vida y su propósito, y tiene el poder de dar forma y significado al ser, saber y hacer, que se puede reconocer como un impulso unificador, un sistema de guía interno básico para el bienestar humano, que motiva para escoger las relaciones y búsquedas necesarias". La enfermedad, con frecuencia, constituye una oportunidad para replantear la vida, para resaltar lo que realmente resulta valioso para la persona, para proponerse nuevas metas que enriquecen la existencia e impulsan a la persona para ir más allá, para trascender. Es recomendable reforzar esta dimensión de acuerdo con las características particulares del enfermo, orientándolo hacia personas, grupos o instituciones que le puedan ayudar al respecto. Los seres humanos tienen diversas creencias; algunos se refugian en aspectos internos como los sentimientos, por ello es trascendente el respeto a su fe y dogmas; también las manifestaciones del espíritu son múltiples, incluyen: expresiones artísticas, la dimensión religiosa; algunos consideran fundamentales el amor, la solidaridad, el cultivo de sí mismo a través de la lectura, la unión familiar, la participación en grupos de reflexión, académicos o sociales, entre otras cosas.

Existen otras situaciones de salud en enfermos con diagnósticos difíciles e irreversibles; por ejemplo, cuando se presentan en personas en estado terminal, puede observarse la importancia que adquiere la dimensión espiritual; sucede como si se diera un vuelco a la escala de valores del paciente y su familia en el que se presentan aspectos trascendentales a los que le había dedicado un gran esfuerzo durante la vida, éstos pasan a un segundo plano (el estatus social, los logros profesionales, el trabajo, la apariencia física), dejando el lugar a otros como: abrazar a un ser querido, expresarle sus sentimientos, también otros como recibir un sorbo de agua dado con afecto, pedir perdón y ofrecerlo, admirar la belleza de la naturaleza, escuchar la risa de un niño, percibir la cercanía de las personas, así como su afecto, sentirse amados y, en ocasiones, orar y llorar con ellos, revisar su fe. Si se está convencido de que el cuidado de enfermería es integral, éstos entonces serán también elementos para tener en cuenta en el plan de alta

Por las consideraciones anteriores el plan de alta se planifica desde el momento del ingreso del enfermo, cuando se realiza la valoración de enfermería y a lo largo de su estancia en la institución, de tal manera que responda puntualmente a las necesidades y recursos. Por ello es importante involucrar a la familia, si la persona lo admite o si se trata de personas que no tienen capacidad para tomar decisiones y cuidar por sí mismas de su salud.

\section{SUGERENCIAS}

Se debe entregar por escrito siempre, con letra clara y en un lenguaje comprensible, el plan de alta que incluya los cuidados que necesita la persona ordinariamente, pero también se debe hacer hincapié en los cuidados especializados que se requieren para determinados padecimientos; pero, aun en estos casos, es indispensable complementarlos de acuerdo con la situación y características particulares de cada persona. Cabe destacar que a través del plan de alta se pretende evitar errores; cuando se establezca el cuidado en el hogar, la capacitación debe ser notificada en la hoja de registros de enfermería, haciendo referencia de quiénes proporcionan la capacitación y quiénes la reciben, de esta manera se tiene la certeza que se van a proporcionar cuidados seguros y de calidad en el hogar.

Por las consideraciones anteriores el profesional de enfermería lleva a cabo promoción para la salud y prevención de la enfermedad, estrategias que deben estar presentes a lo largo de todo el proceso de hospitalización que tienen aplicación a nivel individual y grupal. Se debe registrar si el aprendizaje del cuidador es óptimo o deficiente para reforzar las debilidades en la capacitación; esto va a garantizar que el plan de alta favorezca y proporcione cuidados y seguridad al enfermo en el hogar; al momento de ejecutar y evaluarlos queda asentado en un documento legal que debe estar en el expediente clínico para notificar a otras disciplinas, como a los médicos, enfermeros, nutriólogos y trabajadores sociales, que se proporcionó capacitación individualizada a cada enfermo y cuidador, presentando una visión global, rápida y clara del estado del paciente y de los cuidados enfermeros que se precisan.

\section{REFERENCIAS}

1. Pearlman IR. Discharge planning: the team is behing you! Nurs Manag. 2004; 15 (8): 36-38.

2. Caldera K. Exploration of the effect of educational level on the nurse's attitude toward discharge teaching. Journal of Education. 1999; 19 (8): 24-32.

3. Girard N. The case management model of patient care delivery. AORN J. 1994; 60 (3): 403-404, 411.

4. Pinzón-de Salazar L. El derecho a la información en salud. Centro de Investigaciones y Estudios Bioéticos. Manizales: Universidad de Caldas; 1997: 2.

5. Colombia. Ley 266 de 1996 (enero 25). Asociación Nacional de Enfermeras ANEC. Bogotá: 1996.

6. Pinzón, Op. cit. 
7. Johnson M et al. Citado por Sánchez B. En: El Arte y la Ciencia del Cuidado. Grupo de Cuidado, Facultad de Enfermería. Bogotá: Universidad Nacional de Colombia; 2002: 377.

8. Organización Mundial de la Salud. En Washington: Charlene D. Hill. The Joint Commission. E-mail: chill@jointcommission.org. En Ginebra: Agnes Leotsakos. OMS Alianza Mundial para la Seguridad del Paciente. E-mail: leotsakosa@who.int

9. Sánchez B. Habilidades de cuidado, de los cuidadores de personas en situación de enfermedad crónica. En: El Arte y la Ciencia del Cuidado.
Grupo de Cuidado, Facultad de Enfermería. Bogotá: Universidad Nacional de Colombia; 2002: 374.

10. Sánchez B. Dimensión Espiritual del Cuidado en Situaciones de Cronicidad y Muerte. Revista AQUICHAN. 2004; 4 (4): 6.

11. Tobón-Correa O, García-Ospina C. Promoción de la salud, prevención de la enfermedad, atención primaria en salud y plan de atención básica: ¿Qué los acerca? ¿Qué los separa? Revista Hacia la Promoción de la Salud. 2000; 5: 16 